\title{
Avaliação tomográfica da posição condilar em pacientes portadores de mordida cruzada posterior unilateral funcional
}

Tatiana Dantas da Costa*, Mario Sergio Alves Carneiro**, Jonas Capelli Júnior***

\begin{abstract}
Resumo
Objetivo: avaliar a posição dos côndilos no sentido anteroposterior e no sentido lateral, em indivíduos com mordida cruzada posterior funcional, relacionando-a com o lado em que a mordida cruzada se apresentava, e determinar a existência de modificação da posição condilar desses pacientes após a correção da má oclusão. Metodologia: foi utilizada uma amostra de 13 pacientes que apresentavam mordida cruzada posterior unilateral funcional. Esses pacientes realizaram uma tomografia de três cortes laterais de cada ATM e uma de dois cortes frontais de cada ATM, antes e após a correção da mordida cruzada. Para a correção da mordida cruzada, foi empregado um aparelho do tipo Porter. Resultados: não foi encontrada nenhuma posição condilar específica, para os indivíduos com mordida cruzada posterior funcional, e sim uma grande dispersão dos posicionamentos, tanto no sentido anteroposterior como no lateral. Também não foi encontrada uma correlação significativa entre esse posicionamento e o lado da mordida cruzada, em ambos os sentidos analisados. Foi encontrada modificação da posição condilar após o tratamento da mordida cruzada posterior funcional, porém sem um padrão específico.
\end{abstract}

Palavras-chave: Articulação temporomandibular. Má oclusão. Côndilo mandibular.

\section{INTRODUÇÃO}

A mordida cruzada posterior é considerada uma relação anormal, no sentido transversal, de um dente ou grupos de dentes no arco dentário superior, inferior ou em ambos ${ }^{14,29}$. Manifestase, com frequência, unilateralmente, consistindo numa entidade clínica muito particular, em todos os estágios do desenvolvimento da oclusão. A mordida cruzada posterior unilateral tem caráter funcional quando apresenta um desvio mandibular da posição de relação cêntrica para a de máxima intercuspidação habitual e um desvio da linha média para o lado do cruzamento, quando em posição de máxima intercuspidação habitual 1,17,27,29.

A relação entre a mordida cruzada posterior unilateral funcional e o posicionamento assimétrico dos côndilos dentro da fossa glenoide é considerada em vários trabalhos publicados na literatura odontológica. Essa relação ocorreria devido à atresia maxilar, que gera um desvio funcional da

\footnotetext{
* Mestre e professora assistente de Ortodontia da FO-FBDC

** Professor assistente de Radiologia da FO-UERJ.

*** Doutor e livre docente pela Faculdade de Odontologia da UERJ. Professor associado da Faculdade de Odontologia da UERJ.
} 
mandíbula no momento da oclusão, causado por uma interferência dentária. Esse desvio seria uma busca de um maior número de contatos oclusais e também um posicionamento mandibular mais confortável para o paciente ${ }^{5,13,17,28,29}$.

Embora seja um ponto polêmico, a mordida cruzada posterior unilateral funcional pode causar mudanças gradativas na estrutura esquelética e dentária durante a fase de crescimento e interferir no crescimento e desenvolvimento condilar, levando, eventualmente, a assimetrias esqueléticas e problemas na articulação temporomandibular, já que estudos têm relatado que a ATM é uma área suscetível a alterações de natureza funcional ${ }^{6,14,18}$.

Existe um consenso entre os pesquisadores sobre a realização do tratamento precoce da mordida cruzada posterior unilateral, sendo esperado, com isso, que se estabeleça uma oclusão funcional, eliminando as interferências oclusais e o desvio da mandíbula no fechamento. Como consequência, os côndilos mal posicionados podem assumir uma posição simétrica bilateralmente, contudo poucos estudos têm documentado tais mudanças ${ }^{4,10,14,17,25}$.

Portanto, o estudo dos possíveis efeitos da mordida cruzada posterior unilateral funcional nas articulações temporomandibulares das crianças é de grande interesse científico, mesmo porque ainda existem algumas controvérsias nos trabalhos encontrados na literatura.

O objetivo do presente trabalho é determinar a existência de diferença entre a posição condilar dos pacientes com mordida cruzada posterior funcional antes e após a correção da má oclusão, tanto no sentido anteroposterior como no sentido lateral.

\section{MATERIAL E MÉTODOS}

Nesse estudo foi utilizado um total de 52 tomografias das articulações temporomandibulares direita e esquerda de treze crianças, sendo 10 do gênero feminino e 3 do gênero masculino, portadoras de mordida cruzada posterior unilateral funcional: 13 tomografias de três cortes laterais de cada ATM antes do tratamento (T1); 13 depois do tratamento (T2); 13 tomografias de dois cortes frontais de cada ATM antes do tratamento (T1) e 13 depois do tratamento (T2).

Todas as crianças encontravam-se na fase da dentadura mista, com idades entre 8 e 11 anos (média de 9,3 anos) e eram portadoras de mordida cruzada posterior unilateral funcional, envolvendo pelo menos dois dentes posteriores no quadrante ( $1^{\circ}$ molar permanente mais um molar decíduo) e com a linha média desviada, no mínimo, $1 \mathrm{~mm}$ para o lado do cruzamento (Fig. 1).

Foram excluídos da amostra pacientes que apresentassem discrepâncias esqueléticas sagitais e/ou que possuíssem achados significativos no histórico médico ou qualquer histórico de problemas na articulação temporomandibular.

Todas as tomografias das ATMs foram obtidas em um mesmo equipamento de raios $\mathrm{X}$ panorâmico (Orthophos $\mathrm{CD}^{\circledR}$, SIEMENS, Alemanha), utilizado na técnica panorâmica, podendo ser realizados cortes transversais. Esse aparelho panorâmico é microprocessado e realiza imagens com cortes transversais devido ao movimento horizontal linear tomográfico, e as estruturas fora do plano da imagem não aparecem ${ }^{7}$.

Foi realizada a tomografia de três cortes laterais de cada ATM, em vista lateral (Fig. 2), com os dentes em oclusão. A segunda tomada foi a tomografia de dois cortes frontais de cada ATM, em vista posterior (Fig. 3) com os dentes em oclusão.

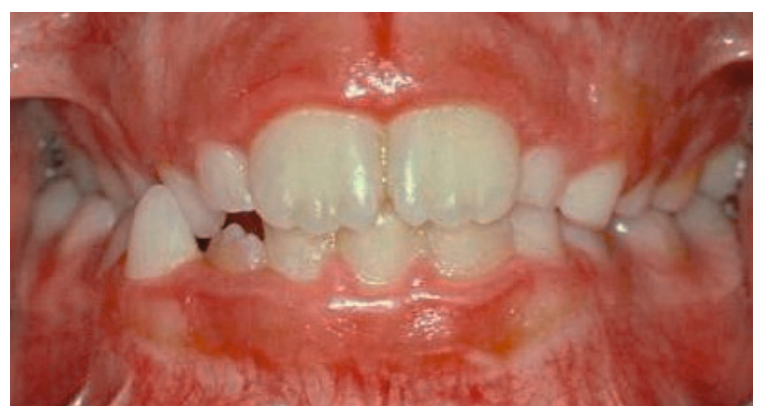

FIGURA 1 - Fotografia intrabucal, vista frontal, destacando a mordida cruzada posterior unilateral com desvio da linha média inferior para o lado do cruzamento. 

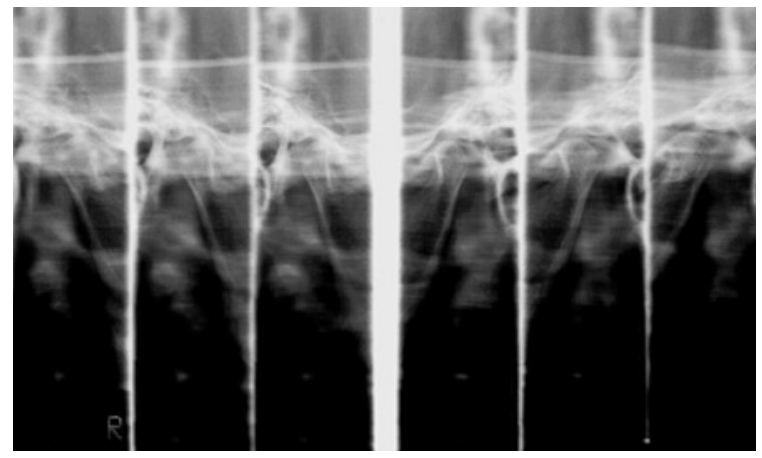

FIGURA 2 - Imagem digitalizada da tomografia de três cortes laterais de cada ATM (vista lateral).

Para que ocorresse uma padronização na realização das tomografias, no mesmo paciente, nos momentos T1 e T2, quando da realização da primeira tomografia (T1) foi preenchida uma ficha, onde foram anotadas as seguintes variáveis: $\mathrm{kV}$, $\mathrm{mA}$, distância do suporte da testa, altura e posição da cabeça, a fim de que essas medidas pudessem ser reproduzidas no mesmo paciente no momento da realização da segunda tomografia (T2) e as duas tomografias, realizadas em momentos diferentes, pudessem ser comparadas.

Os 13 pacientes com mordida cruzada posterior unilateral funcional foram tratados com um aparelho expansor removível tipo Porter. A ativação foi realizada com o arco fora da boca, através da abertura de suas extremidades, o equivalente à largura vestibulolingual dos molares, correspondendo a uma distância de aproximadamente $8 \mathrm{~mm}$ e uma força próxima de $400 \mathrm{~g}$, com intervalo de tempo de seis semanas, continuando até que se conseguisse uma sobrecorreção da mordida cruzada. A partir desse momento, os pacientes permaneceram com o aparelho por um período de seis semanas, a fim de estabilizar a movimentação realizada.

As tomografias foram numeradas aleatoriamente, para que, no momento da realização das medidas, o operador não pudesse identificá-las. As tomografias foram, então, digitalizadas (Scan Jet 4C/T, Hewlett Packard, EUA) a 200dpi (pontos
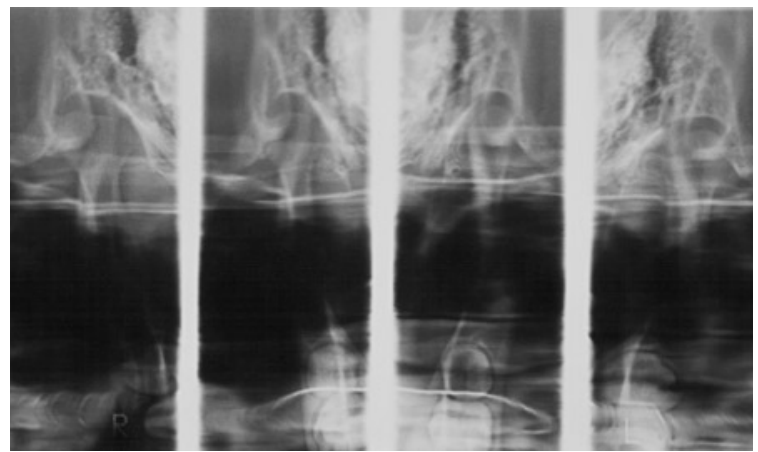

FIGURA 3 - Imagem digitalizada da tomografia de dois cortes frontais das ATMs (vista posterior).

por polegadas). Utilizando o programa Radiocef 2000 versão 3.0 D7 $^{\circledR}$ (Radiomemory, Belo Horizonte/MG), a imagem foi importada e os pontos anatômicos marcados por um mesmo operador. $\mathrm{O}$ programa, então, determinou todos os valores lineares solicitados.

O programa Radiocef possui ferramentas para modificar o contraste, brilho e a escala de cinza, aproximando e detalhando partes da imagem, bem como apresenta o negativo da imagem e uma pseudocoloração. Essas ferramentas foram utilizadas, sempre que necessário, para facilitar a visualização dos pontos anatômicos desejados. A confiabilidade do método foi testada, com baixo erro.

Em ambas as tomografias da ATM foram marcados os pontos de referência, utilizando-se medidas lineares do ponto mais próximo da porção lateral direita e esquerda do espaço articular. A posição condilar relativa, no sentido anteroposterior, foi expressa matematicamente como se segue: o valor do espaço articular posterior $(\mathrm{P})$ menos o valor do espaço articular anterior (A) dividido pelo valor do espaço articular posterior (P) somado ao valor do espaço articular anterior (A), expresso em percentagem [100 x (P-A) / $(\mathrm{P}+\mathrm{A})]$. A localização concêntrica do côndilo na fossa glenoide foi indicada pelo resultado zero, o resultado negativo indicava uma localização posterior e um valor positivo uma localização anterior (Fig. 4), como foi realizado no estudo 


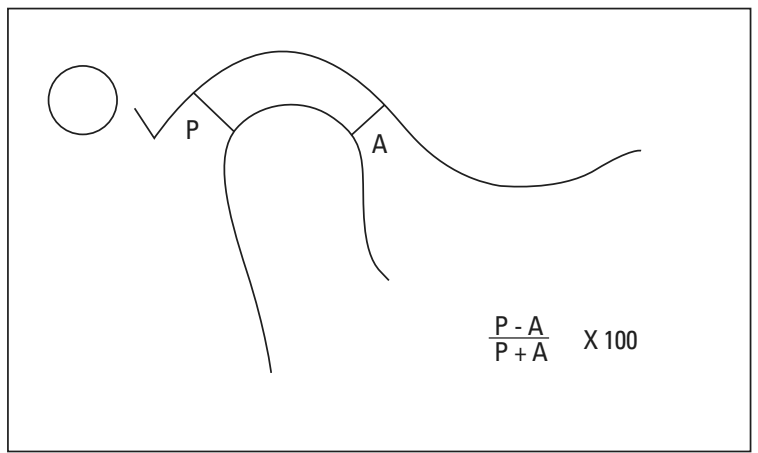

FIGURA 4 - Esquema ilustrativo da medição do espaço articular posterior e anterior.

de Artun, Hollender, Truelove'; Hesse, Artun, Joondeph $^{11}$; Lam, Sadowsky e Omerza ${ }^{15}$; e de acordo com os resultados do trabalho de Pullinger e Hollender ${ }^{24}$.

$\mathrm{Na}$ tomografia das ATMs em vista posterior, com a boca fechada, a posição do côndilo na fossa glenoide foi determinada seguindo o mesmo critério descrito, sendo feita uma adaptação em que o valor do espaço articular do lado direito (D) menos o valor do espaço articular do lado esquerdo (E) dividido pelo valor do espaço articular do lado direito (D) somado ao valor do espaço articular do lado esquerdo (E) resultou em percentagem [100 x (D-E) / (D+E)]. A localização concêntrica do côndilo na fossa glenoide foi indicada pelo resultado zero; o resultado negativo indicava uma localização mais para o lado direito e um valor positivo indicava uma localização mais para o lado esquerdo (Fig. 5) ${ }^{1,11,15,24}$.

O presente estudo foi submetido ao comitê de ética da Universidade do Estado do Rio de Janeiro, que concedeu a autorização para sua realização.

\section{RESULTADOS E DISCUSSÃO}

A escolha da técnica de obtenção da imagem neste estudo foi baseada nas vantagens da tomografia realizada por um aparelho de raios $\mathrm{X}$, quando comparada com a tomografia computadorizada (TC), que são: a utilização de menor dose de radiação $0^{3,30}$, um custo financeiro

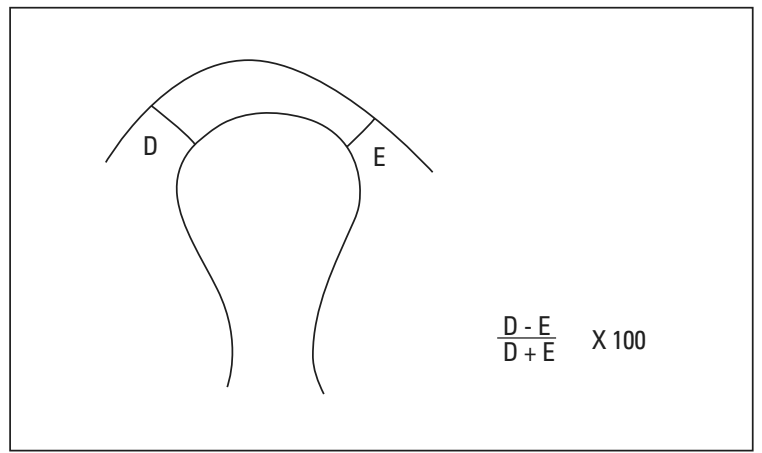

FIGURA 5 - Esquema ilustrativo da medição do espaço articular direito e esquerdo.

consideravelmente menor, em torno de $10 \%$ do valor de uma TC, a facilidade do acesso a esse tipo de imagem ${ }^{7,8}$ e a possibilidade de reprodução da posição da cabeça do paciente e o controle das variáveis durante a tomada radiográfica, o que viabiliza a comparação das tomografias realizadas em diferentes intervalos de tempo. O estudo de Ludlow, Gilbert e Tyndall ${ }^{16}$ também suporta a escolha dessa tomografia, e relata que essa técnica pode ser utilizada como uma alternativa para a tomografia computadorizada.

No presente trabalho, avaliou-se a mudança de posição condilar relativa, tanto no sentido anteroposterior como no sentido transversal de cada ATM, em pacientes com mordida cruzada posterior unilateral funcional, antes e após o tratamento ortodôntico.

No côndilo do lado cruzado, os valores referentes encontrados em $\mathrm{T} 1$ e $\mathrm{T} 2$ foram comparados e analisados estatisticamente, através do teste de Wilcoxon, na tentativa de identificar uma mudança na posição condilar. Porém, não foi encontrado um valor estatisticamente significativo ( $\mathrm{p}>0,05)$ no sentido anteroposterior.

No gráfico 1, pode-se observar o comportamento dos côndilos do lado cruzado (no sentido anteroposterior) dos 13 pacientes da amostra, antes (T1) e depois do tratamento (T2).

Visualmente, fica evidente a grande diferença entre as mudanças de posição condilar relativa de 


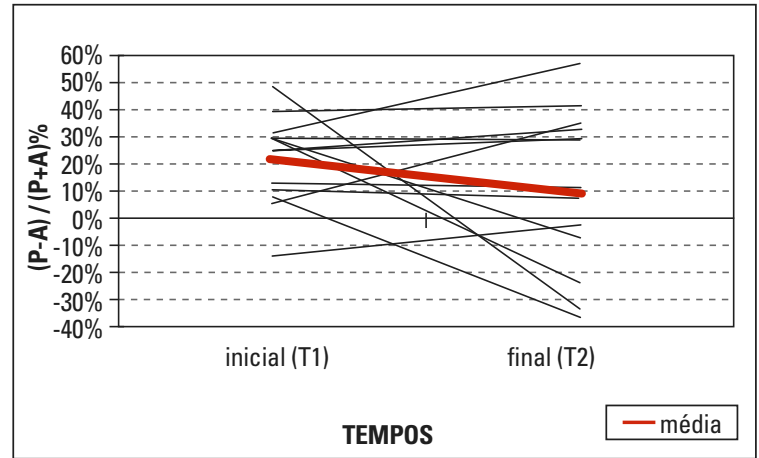

GRÁFICO 1 - Comportamento no sentido anteroposterior dos côndilos do lado cruzado, antes (T1) e depois do tratamento (T2).

T1 para T2. Quanto às mudanças ocorridas após o tratamento, nos côndilos do lado não-cruzado, no sentido anteroposterior, também não foi encontrado um valor estatisticamente significativo ( $p>0,05)$.

É importante ressaltar que com o tratamento, no côndilo do lado não-cruzado, no sentido anteroposterior, foi encontrado um $\mathrm{p}$-valor, pelo teste de Wilcoxon, mais baixo $(0,087)$, indicando uma modificação mais homogênea da amostra entre o posicionamento em T1 para T2. Essa mudança ocorreu de um posicionamento mais anterior $(17,2 \pm 27,8)$ para uma posição mais próxima do centro da fossa glenoide em T2 $(5,7 \pm 26,5)$. Nessa comparação, o desvio-padrão se manteve com valores muito próximos.

O comportamento dos côndilos do lado nãocruzado no sentido anteroposterior dos 13 pacientes da amostra após o tratamento pode ser observado no gráfico 2 . Aqui, também, fica visualmente claro que as mudanças ocorridas no lado não-cruzado foram relativamente mais homogêneas, de $\mathrm{Tl}$ para T2, do que no lado cruzado.

A análise estatística sobre a mudança de posição no sentido transversal de T1 para T2 dos côndilos do lado direito e do lado esquerdo dos 13 pacientes da amostra também não apresentou um resultado estatisticamente significativo ( $p>0,05)$.

Existem alguns trabalhos, que estudaram as modificações do côndilo no sentido anteroposterior

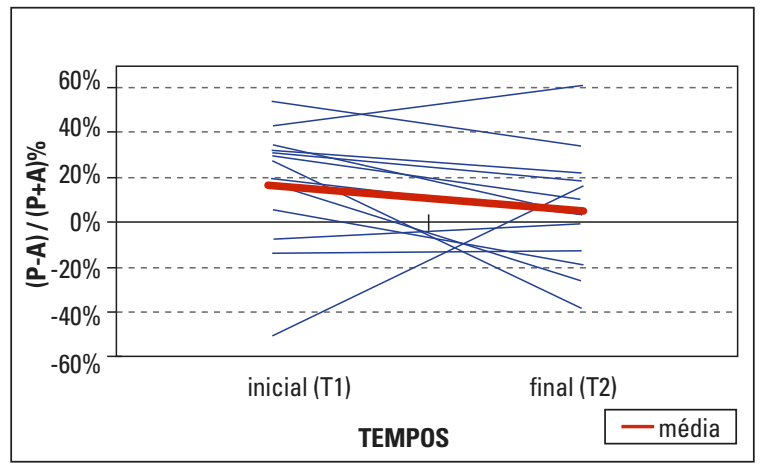

GRÁFICO 2 - Comportamento dos côndilos no sentido anteroposterior do lado não-cruzado, antes (T1) e depois do tratamento (T2).

dentro da fossa glenoide, que também não encontraram alteração da posição condilar, antes e depois do tratamento. Por exemplo, o trabalho de Nerder, Bakke e Solow ${ }^{20}$, que avaliou a posição condilar em 6 crianças com mordida cruzada posterior unilateral funcional (MCPUF), antes e depois do tratamento. Seus resultados demonstraram que os pacientes em oclusão antes do tratamento apresentavam os côndilos numa posição concêntrica. Após o tratamento da MCPUF, a posição condilar dos pacientes em oclusão continuou numa posição normal (concêntrica).

Já no trabalho de Lam, Sadowsky e Omerza ${ }^{15}$, os resultados demonstraram um grande desviopadrão, resultando numa incapacidade de detectar qualquer diferença significativa entre as posições condilares antes e depois do tratamento. Esse desvio-padrão alto indica também uma grande variabilidade na posição condilar em ambos os grupos.

No presente trabalho, o desvio-padrão da diferença da posição condilar relativa antes e depois do tratamento, no sentido anteroposterior, ficou entre $31,4 \%$ e $32,6 \%$, que foi um pouco maior do que o encontrado no trabalho de Lam, Sadowsky e Omerza $^{15}$, que variou de $16 \%$ a $23 \%$, e também no trabalho de Hesse, Artun e Joondeph ${ }^{11}$, que variou de $16 \%$ a $20 \%$.

Apesar das mudanças de posição do côndilo de $\mathrm{T} 1$ para $\mathrm{T} 2$ não apresentarem valores 
estatisticamente significativos no presente estudo, o côndilo do lado não-cruzado foi o que apresentou um p-valor menor $(0,087)$, indicando uma maior similaridade das mudanças ocorridas. A mudança observada no côndilo do lado não-cruzado foi de um posicionamento mais anterior para um posicionamento mais concêntrico, ou seja, o côndilo do lado não-cruzado moveu-se posteriormente. Esse achado concorda com outros relatos encontrados na revisão da literatura.

Os estudos de Myers, Barenie, Bell et al. ${ }^{19}$; Hesse, Artun, Joondeph ${ }^{11}$; Nerder, Bakke e Solow ${ }^{20}$ não demonstraram modificação da posição condilar no lado da mordida cruzada, após seu tratamento, provavelmente devido à maior proximidade desse côndilo ao centro de rotação do movimento mandibular. Indicando, assim, que o côndilo do lado cruzado já estava adequadamente posicionado antes do tratamento. Por outro lado, os resultados do estudo de Santos-Pinto et al. ${ }^{27}$ sugeriram que o tratamento da MCPUF altera a posição de ambos os côndilos, pois os espaços articulares posterior e superior do lado não-cruzado diminuíram, enquanto no lado cruzado estes espaços aumentaram. Esse estudo demonstrou ainda que, após o tratamento, o côndilo do lado cruzado girou anterior e medialmente, enquanto, no lado não-cruzado, o côndilo girou lateral e posteriormente ${ }^{21}$.

Existem, ainda, alguns fatores que também devem ser considerados quando se realiza estudos sobre a posição condilar relativa, como a existência de uma grande variação na posição condilar de uma pessoa para outra e também de um côndilo para outro na mesma pessoa. Além disso, Pandis, Karpac, Trevino ${ }^{22}$; Cohlmia, Ghosh e Sinha et al. ${ }^{9}$ concluíram que a imagem do relacionamento entre o côndilo e a fossa glenoide pode ser afetada pela forma da cabeça do côndilo, especialmente pela forma côncava, onde o côndilo aparece deslocado posteriormente na fossa, não como resultado do deslocamento real, mas como resultado da técnica radiográfica e da morfologia condilar.

Essas divergências entre os resultados obtidos nesses diferentes trabalhos podem estar associadas às diferenças existentes entre o tamanho das amostras, as faixas etárias das crianças, dificuldade no diagnóstico da MCPF, período de contenção para a realização da tomada após o tratamento e os métodos de obtenção de imagem utilizados.

Por se tratar de um estudo longitudinal e pelo tempo disponível para a realização da pesquisa, o presente estudo possui algumas limitações metodológicas inerentes: o tamanho da amostra (13 pacientes) pode ser considerado pequeno e, em virtude da preocupação com a ética da exposição à radiação, não foi utilizado um grupo controle. Essas dificuldades também podem ser observadas no trabalho de Myers, Barenie e Bell et al. ${ }^{19}$, que utilizaram uma amostra de 10 pacientes, sem grupo controle; Nerder, Bakke e Solow ${ }^{20}$, com 6 crianças sem grupo controle; e Santos-Pinto et al. ${ }^{27}$, com 15 pacientes, também sem grupo controle.

Apesar de o presente estudo não ter encontrado nenhuma assimetria na posição condilar antes do tratamento e nenhuma mudança após o tratamento, parece ser válida a orientação do tratamento precoce da MCPUF, porque a autocorreção é improvável, além do fato de que uma eventual assimetria na posição condilar pode predispor a uma compensação assimétrica do relacionamento do côndilo com a fossa, resultando em uma assimetria facial $1^{5,14,17,21,23}$ e aumentando o risco de desordens temporomandibulares ${ }^{32}$. Vários autores têm demonstrado um aumento na atividade eletromiográfica dos músculos da mastigação no lado da mordida cruzada em crianças com desvio mandibular funcional, o que poderia afetar o desenvolvimento mandibular ${ }^{12,31}$.

Existem poucas informações disponíveis sobre a posição condilar normal em crianças, porém a simetria condilar bilateral tem sido associada à ausência de sintomas clínicos em adultos ${ }^{32}$. Por isso, outros estudos devem ser realizados para investigar a posição condilar normal em crianças e o efeito da mordida cruzada posterior funcional no crescimento e desenvolvimento condilar. 


\section{CONCLUSÃO}

Com base nos resultados obtidos, pode-se concluir que foram encontradas modificações na posição condilar, após o tratamento da mordida cruzada posterior unilateral funcional, porém sem um padrão específico. Observa-se, também, que não foi encontrada nenhuma posição condilar específica, para os indivíduos com mordida cruzada posterior unilateral funcional, e sim uma grande dispersão dos posicionamentos, tanto no sentido anteroposterior como no lateral. Também não foi encontrada uma correlação significativa entre esse posicionamento do côndilo e o lado da mordida cruzada, em ambos os sentidos estudados.

\title{
Condylar position tomographic evaluation in patients with functional unilateral posterior cross-bite
}

\begin{abstract}
Aim: To evaluate the condylar position, in anteroposterior and transverse directions, in subjects with functional unilateral posterior cross-bite, relating it to the side in which the cross-bite was present and also to determine if there was any change in the condylar position of these patients after the malocclusion treatment. Methods: It was used a sample of thirteen (13) patients who presented unilateral posterior cross-bite. These patients were submitted to a tomography with three lateral cross-sections of each ATM and another tomography with two frontal cross-sections of each ATM, before and after the cross-bite correction. To treat the cross-bite was used a Porter appliance. Results: It was not found any specific condylar position for the subjects with unilateral posterior cross-bite but a wide variability of positions, not only in the anteroposterior direction but also in the transverse one, it was not found, as well, a significative correlation between the position and the side of the cross-bite in both directions. It was found a change in the condylar position after the treatment of the unilateral posterior cross-bite, however it didn't present a specific pattern.
\end{abstract}

Keywords: Temporomandibular joint. Malocclusion. Mandibular condyle.

\section{REFERÊNCIAS}

1. ARTUN, J.; HOLLENDER, L. G.; TRUELOVE, E. L. Relationship between orthodontic treatment, condylar position, and internal derangement in the temporomandibular joint. Am. J. Orthod. Dentofacial Orthop., St. Louis, v. 101, no. 1, p. 48-53, Jan. 1992.

2. ASSOCIAÇÃO BRASILEIRA DE NORMAS TÉCNICAS. NBR 6023: Informação e documentação - referências - elaboração. Rio de Janeiro, ago. 2000.

3. BEDNAR, J. TMJ imaging. CDS Rev., Chicago, v. 83 , no. 4, p. 45-48, May 1990.

4. BELL, R. A.; LECOMPTE, E. J. The effects of maxillary expansion using a quad-helix appliance during the deciduous and mixed dentitions. Am. J. Orthod., St. Louis, v. 79, no. 2, p. 152-160, Feb. 1981.

5. BISHARA, S. E.; BURKEY, P. S.; KHAROUF, J. G. Dental and facial asymmetries: a review. Angle Orthod., Appleton, v. 64, no. 2, p. 89-98, 1994
6. CAPELOZZA FILHO, L.; SILVA FILHO, O. G. Expansão rápida da maxila: considerações gerais e aplicação clínica. In: INTERLANDI, S. (Org.). Ortodontia: bases para iniciação. 3. ed. São Paulo: Artes Médicas, 1994. p. 393-418.

7. CHEN, S. K.; HOLLENDER, L. Frequency domain analysis of cross-sectional images of the posterior mandible. Oral Surg. Oral Med. Oral Pathol., St. Louis, v. 77, no. 3, p. 290-295, Mar. 1994.

8. CHEN, S. K.; HOLLENDER, L. Linear unsharp mask filtering of linear cross-sectional tomograms of posterior mandible. Swed. Dent. J., Jönköping, v. 19, no. 4, p. 139-147, 1995.

9. COHLMIA, J. T.; GHOSH, J.; SINHA, P. K. et al. Tomographic assessment of temporomandibular joints in patients with malocclusion. Angle Orthod., Appleton, v. 66, no. 1, p. 27-35, 1996.

10. DE BOER, M.; STEENKS, M. H. Functional unilateral posterior crossbite: orthodontic and functional aspects. J. Oral Rehabil. Oxford, v. 24, no. 8, p. 614-623, 1997. 
11. HESSE, K. L.:ARTUN, J.; JOONDEPH, D. R. Changes in condylar position and occlusion associated with maxillary expansion for correction of functional unilateral posterior crossbite. Am. J. Orthod. Dentofacial. Orthop., St. Louis, v. 111, no. 4, p. 410-418, Apr. 1997.

12. INGERVALL, B.; THILANDER, B. Activity of temporal and masseter muscles in children with a lateral forced bite. Angle Orthod., Appleton, v. 45, no. 4, p. 249-258, Oct. 1975.

13. KING, D. L. Functional posterior crossbite in the deciduous and early mixed dentition. Gen. Dent., Chicago, v. 26, no. 1 p. 36-40, 1978

14. KUTIN, G. HAWES, R. R. Posterior crossbites in the deciduous and mixed dentitions. Am. J. Orthod., St. Louis, v. 56, no. 5, p. 491-504, Nov. 1969

15. LAM, P. H.: SADOWSKY, C: OMERZA, F. Mandibular asymmetry and condylar position in children with unilatera posterior crossbite. Am. J. Orthod. Dentofacial Orthop. St. Louis, v. 115, no. 5 , p. 569-575, May 1999

16. LUDLOW, J.; GILBERT, D. B.; TYNDALL, D. A. et al. Analysis of condylar position change on digitally subtracted Orthophos P-4 and Sectograph zonogram images. Int. J. Adult Orthodon. Orthognath. Surg., Chicago, v. 10, no. 3, p. 201-209, 1995.

17. McDONALD, R. E.; AVERY, D. R. Odontopediatria. 4. ed. Rio de Janeiro: Guanabara Koogan, 1986.

18. MOFFETT, B. The morphogenesis of temporomandibular joint. Am. J. Orthod., St. Louis, v. 64, no. 1, p. 401-415, June 1966.

19. MYERS, D. R.; BARENIE, J. T.; BELL, R. A. et al. Condylar position in children with functional posterior crossbites: before and after crossbite correction. Pediatr. Dent., Chicago, v. 2 , no. 3, p. 190-194, 1980.

20. NERDER, P. H.; BAKKE, M.; SOLOW, B. The functional shift of the mandible in unilateral posterior crossbite and the adaptation of the temporomandibular joints: a pilot study. Eur. J. Orthod., Oxford, v. 21, no. 2, p. 155-166, Apr. 1999.

21. O'BYRN, B. L.; SADOWSKY, C.; SCHNEIDER, B. et al. An evaluation of mandibular asymmetry in adults with unilateral posterior crossbite. Am. J. Orthod. Dentofacial. Orthop. St. Louis, v. 107, no. 4, p. 394-400, Apr. 1995.

22. PANDIS, N.; KARPAC, J.; TREVINO, R. et al. A radiographic study of condyle position at various depths of cut in dry skulls with axially corrected lateral tomograms. Am. J. Orthod. Dentofacial Orthop., St. Louis, v. 100, no. 2, p. 116-122, Aug. 1991.
23. PIRTTINIEMI, P. KANTOMAA, T. LAHTELA, P. Relationship between craniofacial and condyle path asymmetry in unilateral cross-bite patients. Eur. J. Orthod., Oxford, v. 12, no. 4 p. 408-413, 1990.

24. PULLINGER, A.; HOLLENDER, L. Variation in condyle-fossa relationships according to different methods of evaluation in tomograms. Oral Surg. Oral Med. Oral Pathol., St. Louis, v. 62, no. 6, p. 719-727, Dec. 1986.

25. PURCELL, P. D. Effectiveness of posterior crossbite correction during the mixed dentition. J. Pedod., Birmingham, v. 9, no. 4 p. 302-311, 1985.

26. SANTOS-PINTO, A. Alterações condilares e dentofaciais em crianças com mordida cruzada posterior unilateral na fase da dentadura mista. 1991. 106f. Tese (Doutorado em Odontologia)-Faculdade de Odontologia da Universidade Federal do Rio de Janeiro, Rio de Janeiro, 1991.

27. SANTOS-PINTO, A.; BUSCHANG, P. H.; THROCKMORTON, G. S.; CHEN, P. Morphological and positional asymmetries of young children with functional unilateral posterior crossbite. Am. J. Orthod. Dentofacial Orthop., St. Louis, v. 120, no. 5, p. 513-520, Nov. 2001

28. SCHRUDER, U.; SCHRUDER, I. Early treatment of unilateral posterior crossbite in children with bilaterally contracted maxillae. Eur. J. Orthod., Oxford, v. 6, no. 1, p. 65-69, 1984.

29. SILVA FILHO, O. G.; SANTAMARIA JÚNIOR, M.; CAPELOZZA FILHO, L. Epidemiology of posterior crossbite in the primary dentition. J. Clin. Pediatr. Dent., Birmingham, v. 32, p. 73-78, 2007

30. STUANI, B.; TELLES, C. S. Imagem por ressonância magnética (IRM) no diagnóstico da ATM. Rev. SBO, Rio de Janeiro, v. 2, n. 8, p. 272-280, jul./dez. 1995

31. TROELSTRUP, B.; MOLLER, E. Electromyography of the temporalis and masseter muscles in children with unilatera cross-bite. Scand. J. Dent. Res., Copenhagen, v. 78, no. 5, p. 425-430, Aug. 1970.

32. WEINBERG, L. A. Technique for temporomandibular joint radiographs. J. Prosthet. Dent., St. Louis, v. 28, no. 3, p. 284-308, Sept. 1972.
Endereço para correspondência

Tatiana Dantas da Costa

Av. Antônio Carlos Magalhães, 585, Ed. Pierre Fauchard, sl. 1206

CEP: 41.825-000 - Salvador / BA

E-mail: tatianadantas@uol.com.br 